\title{
Stereotactic Accelerated Partial Breast Irradiation
}

National Cancer Institute

\section{Source}

National Cancer Institute. Stereotactic Accelerated Partial Breast Irradiation. NCI

Thesaurus. Code C162159.

Partial breast irradiation using real-time tracking, respiratory motion management, and submillimeter accuracy. It results in a reduced target and treatment uncertainty allowing for treatment intensification and maximal target coverage while protecting normal breast tissue from unnecessary high-dose irradiation. 